Recepción: 20 / 04 / 2017

Aceptación: 20 / 05 / 2017

Publicación: 15 / 06 / 2017

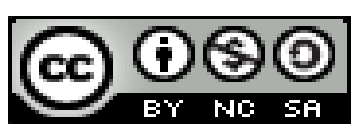

Ciencias Productivas

Artículo de Investigación

\title{
Elaboración de papel vegetal que cumpla las normas TAPPI para el diseño e impresión a partir de la fibra de la cascara del plátano verde
}

\author{
Elaboration of vegetal paper that complies with the TAPPI standards for the design \\ and printing from the fiber of the shell of the green banana
}
Papel TAPPI planta de transformação que satisfaça as normas para a concepção e impressão de fibra de casca de banana verde.

Felix E. Jaramillo-Valle ${ }^{\mathrm{I}}$ felix.jaramillov@ug.edu.ec

Andrea Y. Corral-Ruiz II andrea.corralr@ug.edu.ec

\author{
Maria Milagros de Jesus Fois-Lugo III \\ maria.foisl@ug.edu.ec \\ Brick L. Reyes-Pincay ${ }^{\text {IV }}$ \\ brick.reyesp@ug.edu.ec
}

Correspondencia: felix.jaramillov@ug.edu.ec

\footnotetext{
Docente Contratado de la Carrera de Diseño de Interiores. Facultad de Arquitectura y Urbanismo. Universidad de Guayaquil, Ecuador. Magister en Comunicación Pública de Ciencia y Tecnología - ESPOL; Doctorando en Diseño de la Universidad de Palermo, Buenos Aires, Argentina.

II. Docente contratada de la Carrera Diseño de Interiores; Facultad de Arquitectura y Urbanismo; Universidad de Guayaquil, Ecuador. Máster Universitario en Diseño y Comunicación por la Universidad Pompeu Fabra, Barcelona, España.

III. Docente contratada de la Carrera de Arquitectura; Facultad de Arquitectura y Urbanismo; Universidad de Guayaquil, Ecuador. Master Universitario en Gestion de Proyectos deEdificacion (Project Management); Master Universitario en Planeamiento Urbano y Territorial; Arquitecto, Guayaquil, Ecuador.

Iv. Magister en Impactos Ambientales; Especialista en Estudios de Impactos Ambientales; Arquitecto; Univesidad de Guayaquil, Guayaquil, Ecuador.
} 
Felix E. Jaramillo-Valle; Andrea Y. Corral-Ruiz; Maria Milagros de Jesus Fois-Lugo; Brick L. Reyes-Pincay

\section{Resumen}

En la presente investigación se elaborará un manual para la elaboración de papel vegetal que cumpla las normas TAPPI para el diseño e impresión a partir de la fibra de la cascara del plátano verde ya que estas técnicas antiguas que inusualmente se usan para la elaboración de papel artesanal han funcionado bien debido a que ha sido destinado para impresión serigrafía o manual que no demanden mayor calidad ni parámetros técnicos de su constitución.

Hay que tener en ceunta que la fabricación del papel a mano es una técnica que a pesar de tener miles de años de antigüedad no ha sido explotada adecuadamente y no se consigue papel de buena calidad que pueda reemplazar a los que usamos normalmente.

Muchos artesanos hacen papel a mano sin una técnica científica que permita conocer las propiedades de las fibras a utilizar para elaborar papel para imprenta o impresoras comunes. En nuestro caso elaboraremos papel vegetal a partir de la fibra de la cascara del plátano verde que presente las mejores propiedades de formación física y óptica.

Palabras claves: Cascara de platano verde; normas APPI; papel vegetal; manual; técnicas. 


\begin{abstract}
In the present investigation a manual will be elaborated for the production of vegetal paper that fulfills the TAPPI norms for the design and impression from the fiber of the shell of the green banana since these old techniques that are unusually used for the elaboration of artisanal paper Have worked well because it has been designed for screen printing or manual printing that do not demand higher quality or technical parameters of its constitution.

It must be borne in mind that the manufacture of paper by hand is a technique that despite being thousands of years old has not been properly exploited and paper of good quality that can replace the ones we normally use is not obtained.

Many craftsmen make paper by hand without a scientific technique that allows to know the properties of the fibers to be used to make paper for printers or common printers. In our case we will prepare vegetal paper from the fiber of the shell of the green banana that presents the best properties of physical and optical formation.
\end{abstract}

Key words: Green banana peel; APPI standards; Vegetable paper; manual; Techniques. 
Felix E. Jaramillo-Valle; Andrea Y. Corral-Ruiz; Maria Milagros de Jesus Fois-Lugo; Brick L. Reyes-Pincay

\section{Resumo}

Nesta pesquisa um manual para a produção de papel vegetal que cumpre as normas TAPPI para criação e impressão a partir de casca de fibra de banana verde será desenvolvido como essas técnicas antigas invulgarmente usados para fazer papel artesanal eles têm trabalhado bem porque ele foi destinado para a impressão da tela ou impressão manual não exigir maior qualidade e parâmetros técnicos de sua constituição.

Ceunta deve estar na fabricação de papel mão é uma técnica que, apesar de ter milhares de anos de idade não foi adequadamente explorado e não papel de boa qualidade que podem substituir aqueles que usamos normalmente alcançado.

Muitos artesãos fazem papel artesanal, sem uma técnica científica para conhecer as propriedades das fibras usadas para fazer papel para impressão ou impressoras comuns. No nosso caso papel elaborado a partir de fibra vegetal casca de banana verde apresentar as melhores propriedades e treinamento físico óptico.

Palavras-chave: Casca de banana verde; normas APPI; papel vegetal; manual; técnicas. 


\section{Introducción.}

Por siglos se ha elaborado papel a partir de fibras vegetales y animales, estas técnicas antiguas que hasta hoy se usan para la elaboración de papel artesanal han funcionado bastante bien para pequeñas cantidades de papel destinado a la impresión serigrafía o manual que no demanden mayor calidad ni parámetros técnicos de su constitución. Hasta el siglo XVII fue una labor artesanal, (Berastegi, Calvo, Díez, Elso, García, \& García De Jalón, 2008) que no alteraban en ningún caso la estabilidad y salud ecológica de los ecosistemas naturales. Pero en la actualidad con el desarrollo tecnológico y avances de la ciencia, se han creado nuevas demandas del mercado en cuanto a calidad y acabados de productos impresos, se necesita cada vez más papel para diseños artísticos con acabados diferenciados pero a la vez que sean realizados sobre soportes de alta resistencia, duraderos y bajo costo, para ello se necesita tener documentación técnica de las características de la formación del papel hecho a base de fibras vegetales para darle el tratamiento adecuado en los diseños e impresiones del medio grafico. En el país se fabrica papel artesanal en base a fibras vegetales, pero no existen parámetros técnicos que caractericen al papel, además el costo de fabricar el papel es elevado, haciéndolo inasequible a los estudiantes y profesionales de la Universidad. (Villegas Marín \& González Monroy, 2013) (Martínez de las Marías, 2008) (González Báez, 2002) (Melo-Sabogal, Torres-Grisales, \& Torres-Valenzuela, 2015)

En este estudio se ha planteado la fabricación de papel artesanal de fibra de la cascara de plátano (Musa paradisiaca), fabricándolo artesanalmente con criterios científicos verificando sus propiedades físico - ópticas según las normas TAPPI para la mejor uso cotidiano. 
Felix E. Jaramillo-Valle; Andrea Y. Corral-Ruiz; Maria Milagros de Jesus Fois-Lugo; Brick L. Reyes-Pincay

\section{Materiales y métodos.}

\section{Metodología empleada en este experimento}

En este paso del procedimiento hemos realizado tres experimentos variando el tiempo de cocción para de esta forma tener tres muestras diferentes de papel para su análisis y comprobar cual presenta mejores cualidades físico-mecánicas y ópticas.

\section{Materiales utilizados}

- Licuadora

- Bastidores y marcos

- Tinas

- Pellón, papel secante

- Cocina y gas

- Olla grande

- Sosa Cáustica

- Cola blanca

\begin{tabular}{|cll|}
\hline INGREDIENTES & CANTIDAD & FUNCION \\
\hline Sosa Cáustica & 15 onzas & Degradante \\
\hline Agua & 5 litros & Disolvente \\
\hline Cloro & 1 onza & Blanqueador \\
\hline Cola Blanca & 3 onzas & Aglutinante \\
\hline $\begin{array}{c}\text { Cáscara de } \\
\text { plátano }\end{array}$ & 5 libras & Material base \\
& & \\
\hline
\end{tabular}

\section{Ingredientes y cantidades utilizadas}

Tabla 1. Ingredientes utilizados 


\section{Resultados.}

Habiendo concluido con las mediciones tanto físicas - Morfologicas como ópticas del papel de la cascara de plátano verde podemos concluir que según sus propiedades es un papel que está apto para la impresión offset comercial de pliegos, serigrafía y estampados, no asi para la impresión offset de bobina. En cuanto al color del papel dista mucho de la blancura de los papeles tradicionales el color del mismo es ideal para realizar o imprimir considerando como blanco su fondo, realiza una mezcla de colores y combinaciones.

Explicaremos más en detalle a continuación.

1. De las muestras preparadas, P1, P2, P3 donde se varió el tiempo de cocción de la pulpa compuesta por las fibras de la cáscara del plátano, hemos notado que sus características no han variado, básicamente son iguales a excepción de la resistencia a la tensión.

2. El gramaje es un promedio de $130 \mathrm{gr} / \mathrm{m}^{2}$, lo cual lo ubica dentro de los tipos de papel llamados estucados que son de mayor consistencia que los papeles periódicos y bond, pero tampoco está dentro de la categoría de cartulinas, pero se puede considerar una cartulina para tarjetas o cartulina fina, según esta clasificación este papel si se puede utilizar en forma industrial en la impresión offset de pliegos y cualquier otro tipo de técnica de impresión que no sea por bobina.

3. En cuanto al espesor debemos hacer la siguiente comparación 
Felix E. Jaramillo-Valle; Andrea Y. Corral-Ruiz; Maria Milagros de Jesus Fois-Lugo; Brick L. Reyes-Pincay

\begin{tabular}{|c|c|}
\hline Muestra & \multicolumn{1}{c}{$\begin{array}{c}\text { espesor } \\
\mathbf{m m} .\end{array}$} \\
\cline { 1 - 2 } Papel periodico & 0,08 \\
\hline Papel couche & 0,2 \\
\hline $\begin{array}{c}\text { Papel de } \\
\text { plátano }\end{array}$ & 0,31 \\
\hline
\end{tabular}

\section{Tabla 2}

Notamos que el papel vegetal de plátano es mucho más grueso que el papel periódico y el papel couche, esta es una razón principal por lo que no pueden ser utilizados en la impresión offset rotativa o de bobina. Este calibre ya no se podría disminuir en forma artesanal porque depende mucho de la fuerza en que se compacten las fibras, esto se podría conseguir con prensas especiales de fabricación de papel que en estos casos resulta muy costoso para el proceso. Pero si se llegará a lograr bajar el calibre del papel si se podría utilizar en prensas de bobina e incluso en prensas digitales.

4. Se apunta también que el papel es bastante poroso, esto es debido a la forma en que se fabrica el papel, al ser artesanal no se cuenta con la maquinaria necesaria para compactar la fibra, esto lo que haría en la impresión es consumir más tinta y producir una impresión no homogénea en la superficie. El consumo de tinta es mayor y el tiempo de secado se incrementa ya que la tinta en mayor cantidad se queda entre las fibras, tal como lo demuestran las imágenes. 


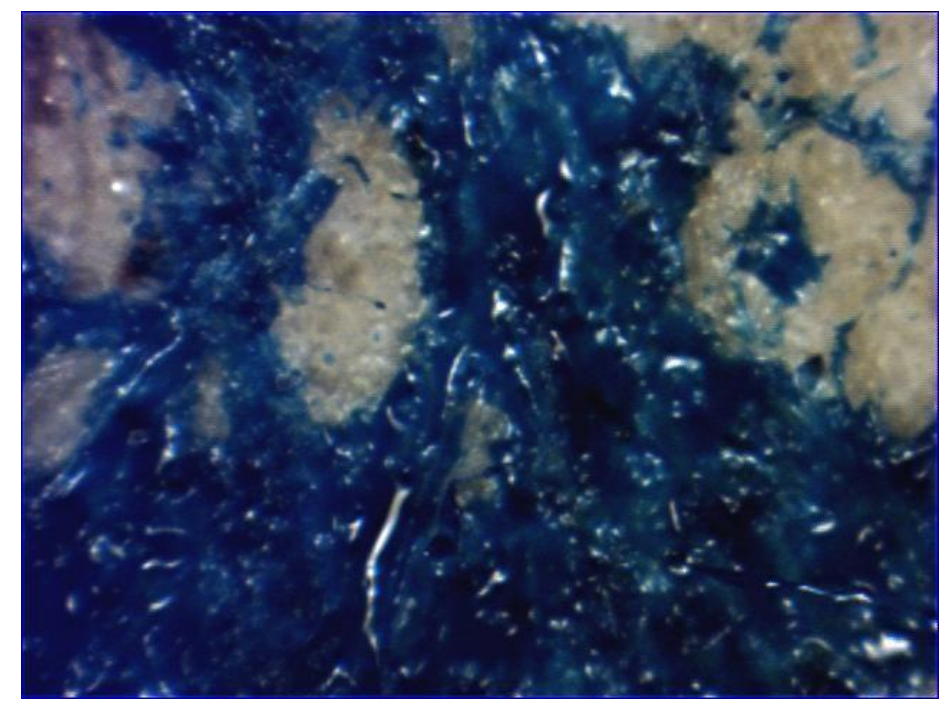

Figura 1. Foto amplificada de tinta sobre el papel color cian

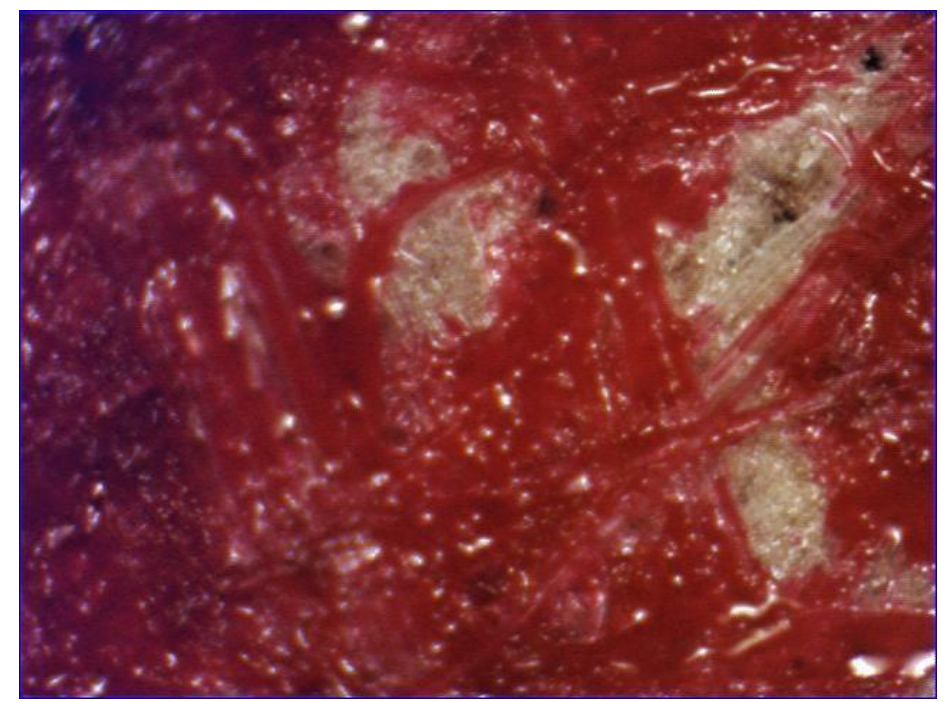

Figura 2. Muestra de tinta sobre papel color magenta

Distribución no homogénea de la tinta sobre la superficie del papel debió a la porosidad y distribución de la fibra.

\section{Rugosidad}

Esta propiedad se la puede evaluar subjetivamente incluso, al pasar la mano y sentir su textura, pues el papel artesanal presenta alta rugosidad producto de la compactación de su fibra. En estos casos en la impresión se necesitaría una mayor densidad de tinta y no es muy favorable para la 
Felix E. Jaramillo-Valle; Andrea Y. Corral-Ruiz; Maria Milagros de Jesus Fois-Lugo; Brick L. Reyes-Pincay

impresión offset, esta es una de las propiedades que se debe mejorar al producirlo en forma industrial. Cabe resaltar que esta textura de los papeles vegetales es un valor agregado ya que es parte de belleza.

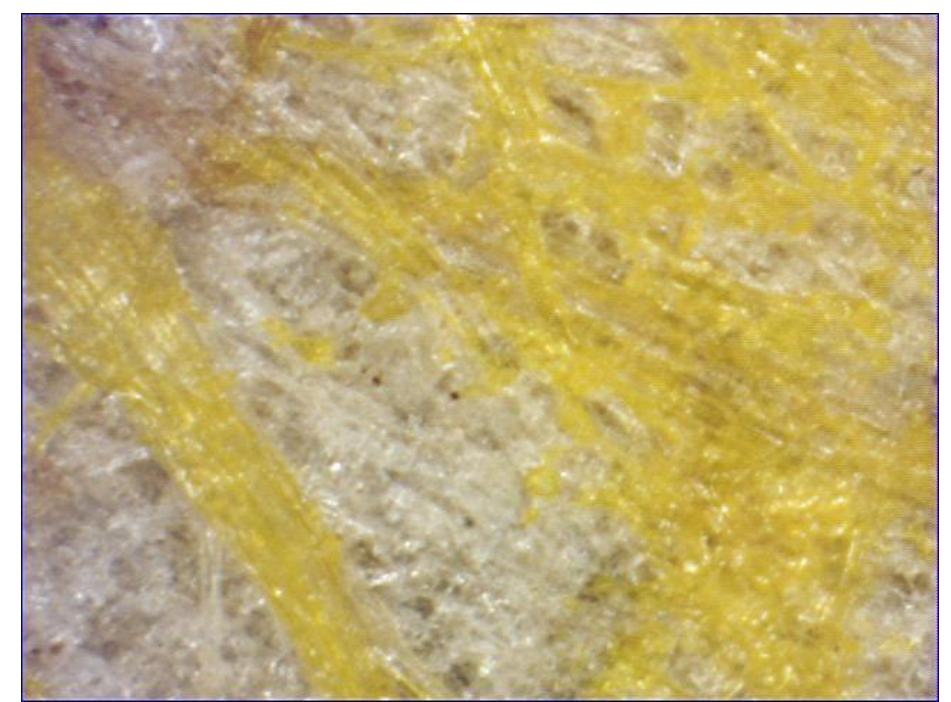

Figura 3. Papel impreso con tinta amarilla donde se nota su aspereza

6. Resistencia a la Tensión, esta es la propiedad que ha permitido romper un tabú en cuanto a la utilización de papel vegetal en la impresión offset. Se dice por muchos años que el papel reciclado no tiene suficiente coeción en sus fibras como para soportar la tensión de arrastre de una prensa. Tal vez el papel reciclado no tenga la fuerza necesaria, pero el papel vegetal de fibra virgen de la cascara de plátano si la tiene.

Y aquí notamos que si existen diferencias entre las diferentes muestras de papel producidas. Las muestras P1 y P3 tienen poca resistencia a la tensión y por lo tanto se romperían en una prensa industrial, pero la muestra P2 que tiene su pulpa un tiempo de cocción intermedio entre las otras dos muestras, presenta una resistencia igual que a la de los otros papeles como son el periódico y bond, haciéndolo idóneo para la impresión offset.

7. Color del papel 
Esta característica nos indica que el papel no cumple ninguna norma por cuanto es demasiado amarillo.

\begin{tabular}{|l|r|r|r|}
\hline \multicolumn{1}{|c}{ L } & \multicolumn{1}{l|}{ a } & b \\
\hline Norma & 82 & 0 & 3 \\
\hline Papel periódico & 88,9 & 1,2 & 2 \\
\hline papel bond & 93 & 2 & $-7,4$ \\
\hline Papel vegetal & 71,7 & 4,7 & 17,5 \\
\hline
\end{tabular}

Tabla 3

Con la explicación anterior sobre el modo de color Lab, notamos que la norma en papel periódico me indica que la blancura es 82 , a igual cero (representa valor verde a rojo) y el valor b es positivo 3 que es amarillo. Los papeles periódicos están dentro del rango de tolerancia a estos valores por que cumplen la norma. El papel bond esta colocado solo como referencia ya que es otro tipo de papel en cuanto a blancura y color, pues su blancura esta sobre los $90 \%$ y su valor b es 7.4 negativo lo que indica que es un papel azulado. El papel vegetal tiene un nivel de blancura de 71.7 bastante por debajo del papel periódico y un valor b muy alto de 17.5, es decir es un papel amarillo con poca iluminación.

Para aumentar la blancura del papel se debe utilizar químicos que aclaren las fibras, pero en este experimento obviamos esto.

El papel vegetal fabricado así, con esa tendencia de color tiene su atractivo, no se lo puede considerar papel blanco pero si un papel artístico que le da otra tonalidad a los colores, pues tiene buen agarre de tinta y crea buenas composiciones de color. 
Felix E. Jaramillo-Valle; Andrea Y. Corral-Ruiz; Maria Milagros de Jesus Fois-Lugo; Brick L. Reyes-Pincay

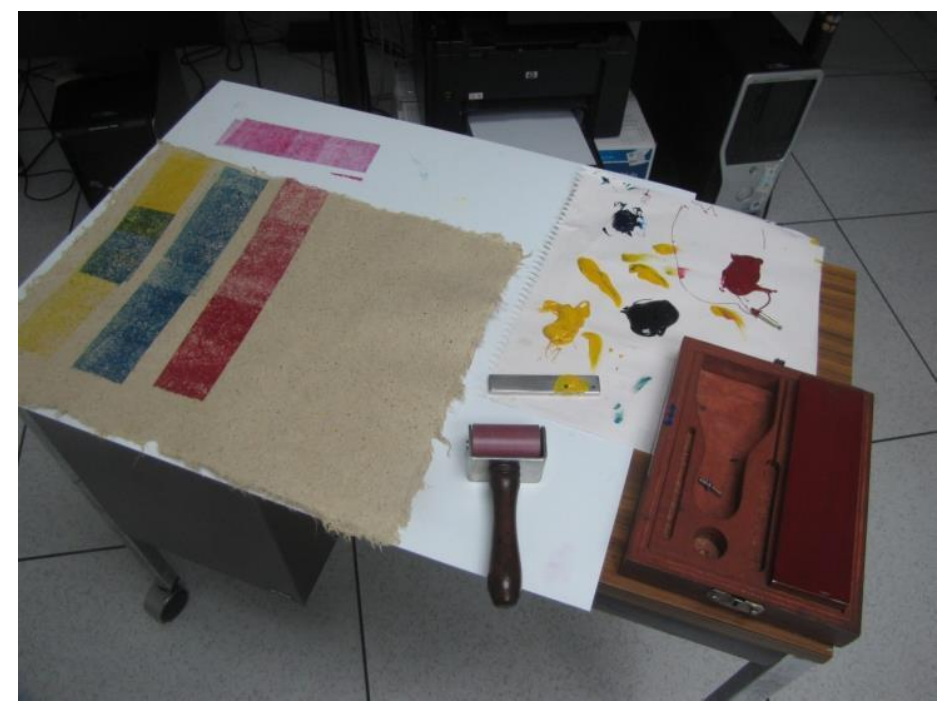

Figura 4. Pruebas de tinta con equipo Quick Pick

\section{Conclusiones.}

Con la obtención de las propiedades del papel de cascara de plátano, concluimos que es un papel tipo estucado de mayor gramaje que los papeles que se utilizan para la impresión offset rotativa como son los papeles periódicos, bond y couche de bajo gramaje, por lo que este papel no puede ser utilizado en la impresión offset convencional, sin embargo presenta propiedades para la impresión offset comercial o prensa plana.

Se concluye también que el papel es bastante poroso, esto es debido a la forma en que se fabrica el papel, al ser artesanal no se cuenta con la maquinaria necesaria para compactar la fibra, esto produce que en la impresión offset comercial se consuma más tinta y producir una impresión no homogénea en la superficie. El consumo de tinta es mayor y el tiempo de secado se incrementa ya que la tinta en mayor cantidad se queda entre las fibras, esto sería su mayor desventaja.

En cuanto al color del papel, el tono amarillo que presenta es su color natural, en estas condiciones concluimos que el papel no cumple ninguna norma colorimétrica por lo que no puede 
Elaboración de papel vegetal que cumpla las normas TAPPI para el diseño e impresión a partir de la fibra de la cascara del plátano verde

ser utilizado para productos corrientes o comerciales a menos que se desee imprimir con esta tonalidad como color base. Pero en contraste a esto el papel puede ser utilizado para impresiones artísticas o que el color del papel sea parte de su diseño general.

El papel puede ser mejorado para su utilización en prensas rotativas y hacerlo incluso más blanco y mejorar su lisura pero sería con la fabricación industrial, de hecho se fabrica papel vegetal para todo tipo de impresión de bajo gramaje y menos amarillos utilizando la tecnología y maquinaria industrial.

\section{Usos del papel.}

De acuerdo a lo tratado en este proyecto este papel de la cascara de plátano tiene múltiples usos de acuerdo a las técnicas de impresión que usemos, podemos utilizarlo en:

\section{Impresión Offset comercial}

La impresión de offset plano convencional es el papel cortado a las medidas de la máquina para imprimir hoja por hoja, si tiene una prensa offset de 8 colores obviamente cortas con la guillotina todos los pliegos de papel a esa medida para que no haya desperdicio. Dependiendo del tamaño y número de cabezas de impresión la maquinaria puedes imprimir desde una nota, factura o pedido hasta un libro, revista o poster tamaño 8 cartas3.

\section{Impresión Serigrafía.}

La serigrafía es una técnica de impresión empleada en el método de reproducción de documentos e imágenes sobre cualquier material, y consiste en transferir una tinta a través de una 
Felix E. Jaramillo-Valle; Andrea Y. Corral-Ruiz; Maria Milagros de Jesus Fois-Lugo; Brick L. Reyes-Pincay

malla tensada en un marco, el paso de la tinta se bloquea en las áreas donde no habrá imagen mediante una emulsión o barniz, quedando libre la zona donde pasará la tinta.4

\section{Impresión Tipografica.}

Este papel también es ideal para la impresión tipográfica, es decir, usando moldes de tipografias se pueden formar frases y textos que luego son estampados en el papel, se pueden realizar muchos tipos de acabados y crear presentaciones muy interesantes.

\section{Impresión láser}

Este tipo de impresión es por calor. La hoja es arrastrada por el interior de la impresora por rodillos calientes que adhieren pigmentos microscópicos de tinta al sustrato.

Pintar a mano, aerografía, acuarela, stencil

Son técnicas manuales de estampación de motivos sobre superficies textiles o sustratos más irregulares que los comunes. Se usan para acabados artísticos o diseño grafico.

Se pueden imprimir productos como:

- Portadas de revistas y libros

- Tarjetas personales

- Invitaciones

- Cuadros con acuarela, plumilla, etc.

- Carteles, letreros. 
- Sobres, carpetas

- Álbumes

- Separadores de libros

- Fundas

- Cajas de regalos.

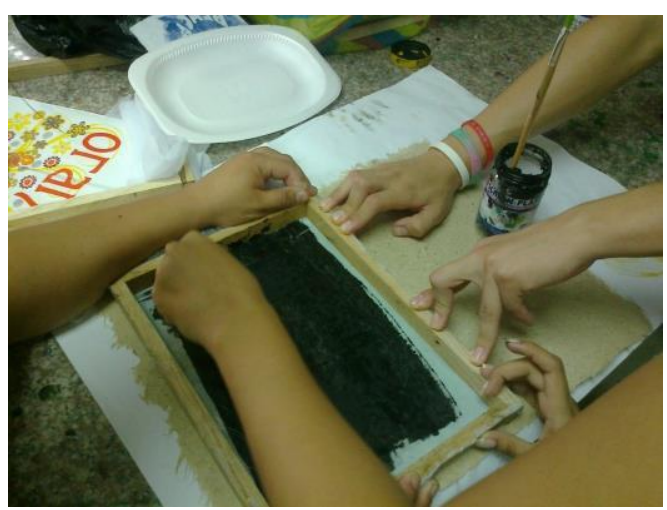

Figura 5. Preparación para la impresión en serigrafía

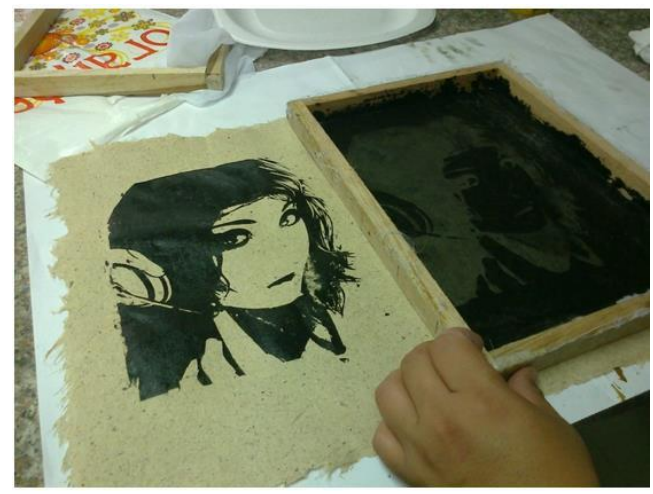

Figura 6. Impresión de rostro en serigrafía 


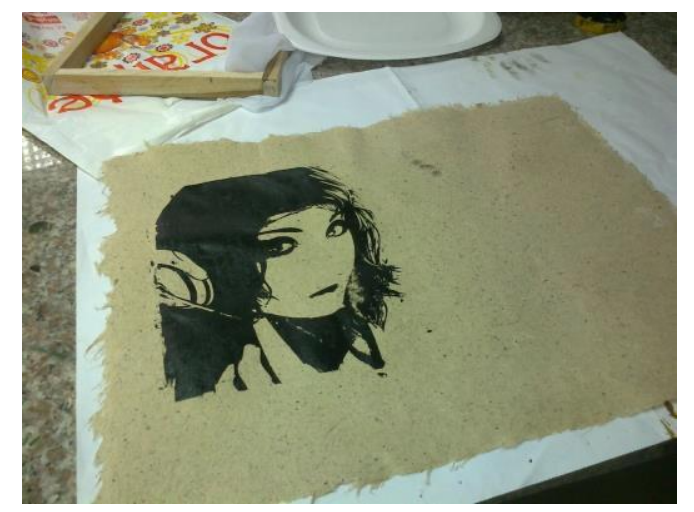

Figura 7. Muestra final de impresión en serigrafía sobre papel vegetal

Este trabajo investigativo busca realizar un importante aporte analítico sobre la importancia y la factibilidad de la fabricación del papel hecho a mano a partir de fibras vegetales de desecho.

Se han explicado las generalidades metodológicas para la fabricación del papel de una manera sencilla y totalmente artesanal sin equipos industriales de alta tecnología.

Se ha fabricado papel de manera menos destructiva pero igual de funcional sin utilizar agentes químicos, su fabricación es más barata y perjudica menos el ambiente. El papel que se ha obtenido es menos blanco pero resistente y más grueso.

Pero se ha demostrado que con estas técnicas de fabricación manual y utilizando la fibra de la cascara del platáneo verde, que la hipótesis principal planteada, en efecto se cumple, pues el papel vegetal de fibra de plátano hecho a mano cumple los estándares de calidad internacionales para ser usado en la industria gráfica industrial como las prensas comerciales de impresión por pliegos y más aún en la impresión serigrafía.

El único valor que no cumple el papel es el referente al color, pues es un papel muy opaco, amarillo con poca luminosidad, pero este color es precisamente el ideal para ser usado en manifestaciones artísticas de creativos, estudiantes y artistas que lo usen. 
Elaboración de papel vegetal que cumpla las normas TAPPI para el diseño e impresión a partir de la fibra de la cascara del plátano verde

El batido es la etapa más importante para lograr papeles de mejores características físicomecánicas, ya que aumenta considerablemente la resistencia a la tensión, además otorga mayor homogeneidad a las hojas de papel y mejora visiblemente su uniformidad.

La elaboración de hojas de papel artesanal depende mucho de la capacitación y la habilidad de quien las fabrica, es por eso que se debe tener cuidado en mantener el espesor y gramaje del papel producido con las medidas ya analizadas que son las mas aceptables para su uso comercial, a esto se debe anotar que cada hoja de papel artesanal es única y diferente.

La fabricación del papel artesanal de papel se podría convertir en una actividad potencial generadora de producción, ingresos y empleo, con efectos multiplicadores sobre los beneficios directos e indirectos. Mejorar la calidad de vida de personas que fabriquen este papel o de los artesanos que ya lo hacen al presentarles otros usos y beneficios de este papel.

\section{Bibliografía.}

Berastegi, A., Calvo, A., Díez, J., Elso, J., García, E., \& García De Jalón, E. (2008). Biodiversidad y restauración de ecosistemas fluviales. Gestión Ambiental, Viveros y Repoblaciones de Navarra. Pamplona: EDICO.

González Báez, C. (2002). Las Fibras Textiles. México: Grupo Radio Centro.

Martínez de las Marías, P. (2008). Física y química de las Àbras textiles. Alhambra: Pearson.

Melo-Sabogal, D., Torres-Grisales, Y., \& Torres-Valenzuela, L. (2015). Aprovechamiento de pulpa y cáscara de plátano (musa paradisiaca spp) para la obtención de maltodextrina. Biotecnología en el Sector Agropecuario y Agroindustrial, 76-85.

Villegas Marín, C., \& González Monroy, B. (2013). Fibras textiles naturales sustentables y nuevos hábitos de consumo. Revista Legado de Arquitectura y Diseño,, 13(1), 31-45. 